\title{
Observational evidence of unwinding and chirality changing in penumbral filaments by Hinode
}

\author{
Jiangtao $\mathrm{Su}^{1,2}$ and $\mathrm{Yu} \mathbf{L i u}^{3}$ \\ ${ }^{1}$ Key Laboratory of Solar Activity, National Astronomical Observatories, \\ Chinese Academy of Science, Beijing 100012, China \\ email: sjt@bao.ac.cn \\ ${ }^{2}$ State Key Laboratory of Space Weather, Chinese Academy \\ of Sciences, Beijing 100190 \\ ${ }^{3}$ National Astronomical Observatories, Yunnan observatory, Chinese Academy of Sciences
}

\begin{abstract}
In this paper, we study twisting motions of the penumbral filaments of active region NOAA 10930. At first, they made unwinding motions. After unwinding, the filaments are found to twist again and developed the opposite chirality in them. These observational results indicate that penumbral filaments are more inclined to be the twisted magnetic flux tubes in nature.
\end{abstract}

Keywords. Sun: Photosphere; Sun: Filaments; Sun: Magnetic fields

\section{Introduction}

Currently, two schematic models are intensely studied on the origin of penumbral filaments, especially for the formation of dark-cored filaments. The first one is the uncombed model which envisages the penumbra as a collection of horizontal flux tubes embedded in a more vertical and stronger background field (Solanki \& Motavon (1993)). In this model, the filaments with dark cores are explained as a consequence of the higher density of the plasma inside the tubes, which will shift the surface of optical depth unity toward higher (cooler) layers (Ruiz Cobo \& Bellot Rubio (2008)). The second one is a field-free gap model (Spruit \& Scharmer (2006)), which envisages the filaments as the consequence of non-magnetized convecting hot gas protruding into the background of oblique magnetic fields. The filaments with dark cores are then explained as a combination of increased opacity associated with a strongly reduced field strength and an overall drop of temperature with height. In this study, we find the filaments in the PIL regions not only unwind but also go further to change their chirality. This dynamical behavior can be easily explained if the penumbral filaments are treated as the twisted flux tubes.

\section{Data analysis and results}

We mainly use the data set of G-band $4305 \AA$ images (GBIs) which were taken by the SOT instruments onboard Hinode. Active region NOAA 10930 consists of a major sunspot (negative polarity) and a minor sunspot (positive polarity). The penumbral filaments of interest were located in between the two sunspots marked by two dashed-frames in panels (a) and (b) of Figure 1. A filament in Frame 1 shows clear right-handed twist, while the one in Frame 2 does not show so clear twist as the first one. However, about 2 hours later, a filament with left-handed twist was found formed there (see Figure 3).

Figure 2 shows time sequence of a penumbral filament in Frame 1 of Figure 1. The filament consists of two slender ones, f1 and f2. They twisted together in the outer penumbra. At 12:48 UT, the lower part of the filament obviously expanded. At 13:15 $\mathrm{UT}$, the profiles of $\mathrm{f} 1$ and $\mathrm{f} 2$ can be discerned possibly due to their counter-clockwise 



Figure 1. GBIs for the southern half of active region NOAA 10930 on 2006 December 10 (a) and $11(\mathrm{~b})$, respectively. The field of view (FOV) is $76 \times 38$ arcsec.
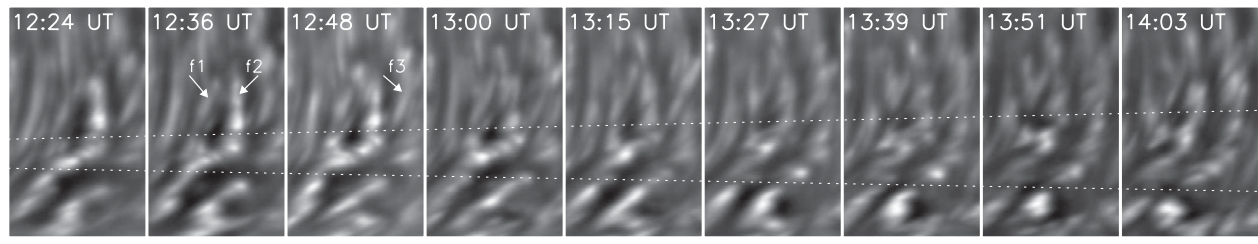

Figure 2. Time sequence of GBIs for an untwisting filament with right-handed chirality on December 10. Its FOV is marked with a dashed-frame in Figure 1(a). The FOV is $6.6 \times 11$ arcsec.
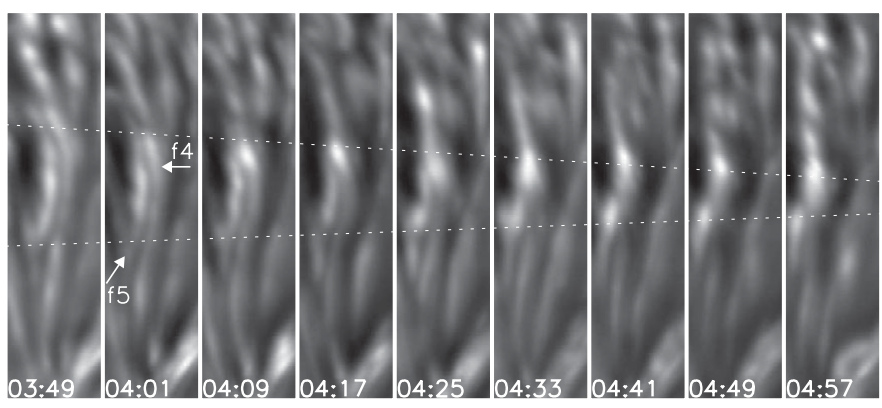

Figure 3. Time sequence of GBIs for the generation of a filament with left-handed chirality on December 11. The FOV is marked with a dashed-frame in Figure 1(b). The FOV is $3.3 \times 13.2$ arcsec.

twisting motions. Similarly, a neighboring filament f3 to the right also underwent such rolling motions. We can trace the shifts of two separating features threaded by two dottedlines at each panel. The top/lower one moved upward/downward at a speed of $\sim 0.31$ / $0.24 \mathrm{~km} \mathrm{~s}^{-1}$. That means the filament underwent untwisting motions in it. Figure 3 shows time sequence of two filaments, f4 and f5, in Frame 2 of Figure 1. At 04:17 UT, they twisted together at the top and a new filament formed. Similarly, we find two converging features in this figure threaded by two dotted-lines at each panel. The top/lower feature moves downward/upward at a speed of $\sim 0.44 / 0.33 \mathrm{~km} \mathrm{~s}^{-1}$. This convergence indicates the filament twisting and shrinking. These dynamical behaviors can be easily explained if the penumbral filaments are treated as the twisted flux tubes.

\section{Acknowledgement}

This work is supported by the Grants: KJCX2-EW-T07, 2011CB811401, 11178005, 10878004, 11221063, 11203036, and the Specialized Research Fund for State Key Laboratories.

\section{References}

Ruiz Cobo, B. \& Bellot Rubio, L. R. 2008, AA, 488, 749

Solanki, S. K. \& Motavon, C. A. P.. 1993, AA, 275, 283

Spruit, H. C. \& Scharmer, G. B. 2006, AA, 447, 343 